\title{
Chapter 16 \\ Design, Implementation, and Field \\ Experimentation of a Long-Lived Multi-hop Sensor Network for Vineyard Monitoring
}

Giuseppe Anastasi, Marco Conti, Mario Di Francesco, and Ilaria Giannetti

\begin{abstract}
Precision agriculture can particularly benefit from wireless sensor networks, as they allow continuous and fine-grained monitoring of environmental data, which can thus be used to reduce management costs and improve crop quality. Such applications typically require long-term and unattended monitoring of large geographical areas. Therefore, sensor nodes must be able to self-organize and use their limited energy budget very efficiently, so as to prolong the network lifetime to many months or, even, years. In this chapter we present ASLEEP, an adaptive strategy for efficient power management in multi-hop WSNs targeted to periodic data collection. The proposed strategy dynamically adjusts the active periods of nodes to match the network demands with the minimum energy expenditure. In this chapter we focus on the implementation and the experimental evaluation of ASLEEP on a real testbed deployed in a vineyard, according to the case study considered in the project. We show that our adaptive approach actually reduces the energy consumption of sensor nodes, thus increasing the network lifetime up to several years.
\end{abstract}

\subsection{Introduction}

A wireless sensor network (WSN) consists of a number of tiny sensor nodes deployed over a geographical area. Each node is a low-power device which is able to sense physical information from the environment (e.g., temperature), process the acquired

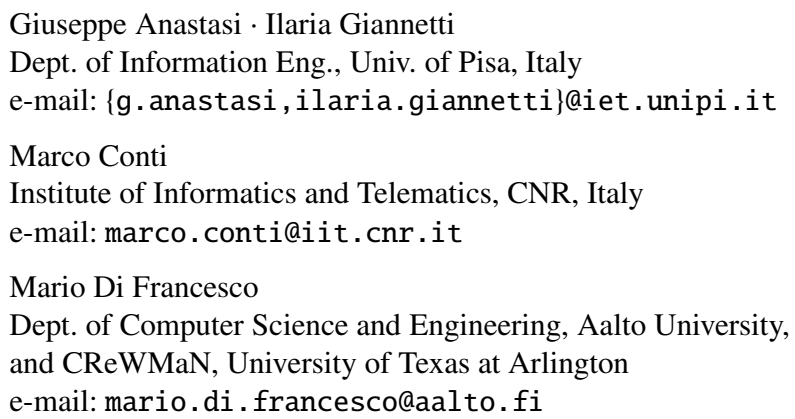


data locally, and send them to one or more collection points, referred to as sinks, base stations [1], or gateways (Chapter 15]). The number of potential WSN applications is extremely large. However, precision agriculture is one application that can particularly benefit from WSNs. In fact, instrumenting crops with wireless sensor nodes enables continuous and fine-grained monitoring of environmental data — such as temperature, humidity, soil moisture - which can be used by farmers to optimise their strategies, reduce management costs, improve quality and, ultimately, increase profits ([31, 5], Chapter 15]. Typically, such applications require monitoring large geographical areas for very long times (e.g., several months, or even years). The key issue is that sensor nodes are generally powered by batteries with a limited capacity and, often, cannot be replaced nor recharged, due to environmental or cost constraints. Therefore, efficient power management strategies should be devised at sensor nodes to extend the network lifetime as much as possible, and avoid frequent battery replacements. Efficient power management is required also when energy can be harvested from the external environment (e.g., through solar cells) (Chapter[15), since a battery has to be used as a buffer anyways.

Since in these applications the radio component accounts for the major energy consumption of a sensor node, even when it is idle [22], the most effective approach to energy conservation is duty-cycling, which consists in putting the radio in the (lowpower) sleep mode during idle periods. Thus, sensor nodes should coordinate their sleep and wakeup periods, and agree on a network-wide sleep schedule in order to make communication feasible and efficient [3]. In the previous chapter a TDMA (Time Division Multiple Access) based duty-cycling scheme was proposed for a star network where all nodes are directly connected to the gateway (Chapter 15). This scheme is very effective for single-hop networks. However, it exhibits limited scalability and flexibility properties in multi-hop sensor networks. In this chapter we focus on multi-hop WSNs, which are required when the monitoring area is large. Most dutycycling schemes for multi-hop WSNs use fixed parameters i.e., the sleep/wakeup periods of different sensor nodes are defined before the deployment and cannot be changed during the operational phase. Fixed duty-cycling schemes require simple coordination mechanisms but, typically, have non-optimal performance. In this chapter we present an Adaptive Staggered sLEEp Protocol (ASLEEP) for multi-hop WSNs, which automatically adjusts the activity of sensor nodes, achieving both low power consumption and low message latency. With respect to other similar approaches, our scheme has two major strengths. First, it is able to quickly adapt the sleep/wakeup periods of each single node to the actual operating conditions (e.g., traffic demand, link quality, node density etc.), resulting in a better utilization of the energy resources and, hence, in a longer network lifetime. In addition, it is not tied to any particular MAC (Medium Access Control) protocol and, thus, it can be used with any sensor platform.

A detailed simulation analysis of ASLEEP has been carried out in [4]. The obtained results have shown that ASLEEP largely outperforms commonly used fixed duty-cycling schemes for multi-hop WSNs in terms of energy efficiency, message latency, and delivery ratio. In this chapter we focus on the implementation of the proposed protocol in actual sensor nodes and its experimental evaluation in a real 
environment. To this end, we deployed a testbed in a vineyard, according to the case study considered in the project, and performed several experiments, in both stationary and dynamic conditions. The experimental measurements confirm previous simulation results, and show that ASLEEP increases significantly the network lifetime, thus making the deployment of a long-lived WSNs really possible.

The remainder of this chapter is organized as follows. Section 16.2 surveys the related work. Section 16.3 introduces the reference system model and outlines the main design principles. Section 16.4 describes the ASLEEP protocol. Section 16.5 introduces the testbed used for the experimental analysis, while Section 16.6presents the obtained results. Finally, Section 16.7 concludes the chapter.

\subsection{Related Work}

Power management can be implemented either at the MAC layer — by integrating a duty-cycling scheme within the MAC protocol - or as an independent sleep/wakeup protocol on top of the MAC layer (e.g., at the network or application layer). Since the solution proposed in this chapter belongs to the latter class, below we will focus on independent sleep/wakeup schemes only.

General sleep/wakeup schemes can be broadly classified into three main categories: on-demand, asynchronous and scheduled rendezvous schemes. On-demand schemes assume that destination nodes can be awakened somehow just before receiving data. To this end, two different radio transceivers are typically used [24, 30]. The first radio (wakeup radio) - typically a very low-power radio — is used to wake up a target node when needed, while the second radio (data radio) is used for the regular data exchange. These schemes can achieve a high energy efficiency and a very low latency. However, they cannot be always used in practice because commonly available sensor platforms only have one radio.

A different option is using an asynchronous scheme [11, 20, 30, 32]. In this case a node can just wakeup whenever it wants and still be able to communicate with its neighbours. Although being robust and easy to implement, asynchronous schemes generally present high latency in message forwarding and are not very suitable to manage broadcast traffic.

The last class of general sleep/wakeup protocols is represented by scheduled rendezvous schemes, which require that sensor nodes are synchronized and nodes that need to communicate wake up at the same time. Our ASLEEP protocol belongs to this category. A possible approach to scheduled wakeups consists in establishing a coarse-grained TDMA schedule defined at the application layer, and exploiting an underlying MAC protocol for actual data transfer. This approach is used by Flexible Power Scheduling (FPS) [8, 9], which includes an on-demand reservation mechanism capable to dynamically adapt to traffic demands. Since slots are relatively large, a strict synchronization among nodes is not required. However, FPS borrows some drawbacks [23] from TDMA schemes, i.e., it has limited scalability and flexibility in adapting to traffic and topology changes in a multi-hop network. 
Most solutions following a scheduled rendezvous approach use a simple dutycycle-based scheme. For instance, the well-known TinyDB query processing system [27] includes a sleep/wakeup scheme based on a fixed duty-cycle. All sensor nodes in the network wake up at the same instant and remain active for a fixed time interval. An improvement over this simple approach is the staggered scheme included in TAG (Tiny AGgregation) [16, which relies on a routing tree rooted at the sink node. In this scheme, the active times of sensor nodes are staggered according to their position in the routing tree. Nodes located at different levels of the routing tree wake up at different, progressive times, like in a pipeline. Due to its nice properties, this scheme has been considered and/or analysed in many subsequent papers $([6,13,14,15,19,12]$ among the others).

Although providing a basic form of adaptation (wakeup times are staggered to the network topology), this scheme is not able to react to varying operating conditions as active times are fixed and equal for all nodes in the networks. This constraint simplifies the coordination among nodes, but results in low energy efficiency and high message latency. Like TAG, our proposal leverages a staggered approach. However, in our proposal nodes' active periods are dynamically adapted to the observed network conditions and can be tailored to the actual needs. By minimizing the active period of each single node, our adaptive protocol significantly increases network lifetime and reduces message latency.

\subsection{Network Model and Design Principles}

We address the problem of data collection in dense WSNs, where nodes are assumed to be static. We also refer to the common convergecast scenario where data typically flow from sensor nodes to the sink, while data in the opposite direction are much less frequent. We assume that nodes are organized to form a logical routing tree (or data gathering tree), rooted at the sink, for data forwarding 1 . The routing tree may change over time due to link/node failures or nodes running out of energy. Also, it might be recomputed periodically to better share energy consumption among nodes. However, as nodes are static, we assume that the routing tree — once established remains stable for a reasonable amount of time. We also assume that sensor readings are periodically reported to the sink node, e.g., every minute. Specifically, nodes share a common notion of time and communicate each other in communication periods that repeat periodically. Each communication period includes an active interval during which nodes are awake and can communicate with their neighbours (by using the underlying MAC protocol), and a silence interval during which nodes turn their radio off to save energy. The active interval can be made of one or more talk intervals, defined as the time shared between a given node and its children. It is wortwhile noting that the active interval is made of only a single talk interval for both the sink (which

\footnotetext{
${ }^{1}$ Many routing protocols for WSNs rely on a routing tree, e.g., [10, 14, 16, 18, 26, 27].
} 
has only its own talk interval with its children) and leaf nodes (which have only the talk interval with their parent).

We referred to the following design principles to effectively design our power management strategy.

- Low-latency and Energy-efficient Operations. Although the duty-cycle mechanism helps to reduce the energy consumtpion, the power management strategy has to be carefully defined, in order to keep overheads (e.g., radio state switching) as low as possible. In addition, the latency of data collection should not be significantly affected by the duty-cycle scheme.

- Adaptive Duty-Cycle. Since the actual time required for transmitting/receiving all data depends on the time-varying operating conditions, the active interval should be adjusted over time. In addition, as sensor nodes at different locations experience different traffic and network conditions, nodes should set the length of the active interval on an individual basis.

- Distributed and Local Computation. The algorithm used by nodes for the active interval calculation should be local and simple. A global algorithm would require the exchange of information among nodes, thus consuming additional energy. In addition, a complex algorithm might not be suitable for devices with limited computational capacity.

- Coordinated and Robust Network-wide Sleep Schedule. A variation in the active interval of a single sensor node should not compromise the correctness and energy efficiency of the global schedule. Hence, a robust cooperation mechanism is required for nodes to manage the network-wide sleep schedule.

In order to address all those principles, we defined the following power management strategy. First of all, each parent node is responsible for choosing its own talk interval with the children. As a consequence, parent nodes can define the most appropriate talk interval duration, depending on their specific needs. The decision is not centralized, hence parent nodes can choose a specific talk interval duration based on local measurements of the current network activity. In addition, to achieve a low latency, the active intervals are staggered according to the position of nodes along the routing tree. Specifically, sensor nodes at a given level in the routing tree wake up earlier than their ancestors, and the active intervals of intermediate nodes span over two adjacent talk intervals (Fig.16.1). In a staggered scheme, latency of messages flowing from source nodes to the sink is bounded by the sum of the talk intervals of traversed nodes, and is independent from the duration of the communication period [12]. In addition, latency can be further reduced if sensor nodes remain active for the minimum time needed for message exchange, i.e., sending and/or receiving all messages addressed to the sink. In order to approximate this behaviour, the power management strategy includes an estimation algorithm such that parent nodes can estimate the actual talk interval they need. Finally, the power management strategy provides a sleep coordination algorithm to organize and update the newtork-wide sleep schedule according to the talk intervals dynamically selected by individual parent nodes. 


\subsection{Protocol Description}

In this section we present the ASLEEP protocol. We will describe first the talk interval prediction algorithm used by each node for estimating its forthcoming talk interval, then the sleep coordination algorithm used to propagate the new sleep schedule throughout the network.

Throughout we will refer to the talk interval shared by a generic node $j$ and (all) its children during the $m$-th communication period $\gamma^{m}$ as $\tau_{j}^{m}$ (Fig.16.1). For convenience, the duration of the talk interval is defined as an integer number of slots, whose duration is set to $q$. In addition, part of the talk interval is reserved to the transmission of control messages. This part is called beacon period, and its duration is denoted as $\beta$.

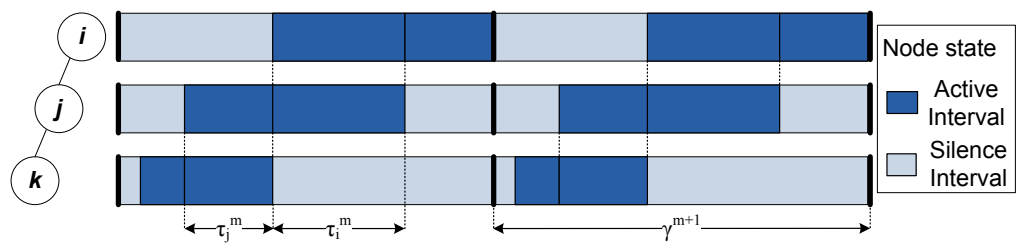

Fig. 16.1 Sleep scheduling parameters

\subsubsection{Talk Interval Prediction Algorithm}

The duration of the talk interval to be shared with the children in the next communication period is dynamically estimated by each parent node. In principle, any algorithm can be used to obtain such an estimate. In order to address the requirements outlined in Section 16.3 we used the algorithm discussed below.

Each parent node measures and stores two values: the message inter-reception time $\left(\Delta_{i}\right)$, i.e., the time elapsed between two consecutive messages which are correctly received in a specific communication period; and the total number of messages $(n)$ correctly received in the same communication period. The time needed to receive all messages sent by children in the next communication period is then estimated as $\bar{\Delta} \cdot N$, where $\bar{\Delta}$ and $N$ are the average inter-reception time and the maximum number of received messages over the observation window made of the last $L$ communication periods, respectively. Using $N$ is a conservative choice to cope with possible message losses. The former time interval is increased so as to accommodate the beacon period. Finally, the expected talk interval is discretized into an integer number of time slots. Hence, the expected talk interval for the $(m+1)$-th communication period can be expressed as $\hat{\tau}^{m+1}=\lceil(\bar{\Delta} \cdot N+\beta) / q\rceil \cdot q$. The duration $q$ of the talk interval slot should be chosen as a trade-off between efficiency and stability. A low value of $q$ allows a fine granularity for the talk interval duration, but may introduce frequent changes in 
the sleep schedule. Conversely, a large value of $q$ makes the schedule more stable, but may lead to talk intervals much larger than necessary, thus wasting energy 2 .

Advertising $\hat{\tau}^{m+1}$ to children as the next talk interval might lead to frequent variations in the schedule parameters of sensor nodes. Hence, the talk interval for the next communication period, $\tau^{m+1}$ is determined as follows. If $\hat{\tau}^{m+1}-\tau^{m}>0$, then $\hat{\tau}^{m+1}$ is chosen as the estimated value and advertised to children (i.e., $\tau^{m+1}=\hat{\tau}^{m+1}$ ). If the predicted talk interval is below the current value and the difference is greater than, or equal to a guard threshold $g$ (i.e., $\tau^{m}-\hat{\tau}^{m+1} \geq g$, with $g \geq 2 q$ ), then the talk interval is decreased by just one time slot $\left(\tau^{m+1}=\tau^{m}-q\right)$. Finally, if $0<\tau^{m}-\hat{\tau}^{m+1}<g$, the talk interval is not immediately decreased. However, if the same condition persists for a number $l$ of consecutive communication periods, then the talk interval is reduced anyway. According to the rules discussed above, an increase in the talk interval is managed less conservatively than a decrease. This is because a more aggressive increase tends to minimize the probability that a node can miss messages from its children.

\subsubsection{Sleep Coordination Algorithm}

Although parent nodes can independently set their talk intervals, a collective effort is needed for the schedule of the whole network to remain consistent and energy efficient. Hence, as a result of a change in the talk interval of a single parent node, the network-wide schedule needs to be re-arranged. This is accomplished by appropriately shifting the active intervals of nodes to ensure that: $i$ ) the active intervals of all nodes are properly staggered; and $i$ ) the two talk intervals of parent nodes are contiguous. Two special messages, direct beacons and reverse beacons, are used for propagating schedule parameters to downstream and upstream nodes, respectively.

Direct beacons are broadcast at each communication period by every parent node at the end of the talk interval, during the beacon period. They include the schedule parameters for the next communication period. Specifically, the direct beacon sent by a node $j$ in the $m$-th communication period contains: $i$ ) the length of the next communication period $\left.\gamma^{m+1} ; i i\right)$ its next wakeup time $t_{\text {parent, }}^{m+1}$; and iii) the length of the next talk interval to be shared with its children $\left(\tau_{j}^{m+1}\right)$.

Conversely, reverse beacons are sent by child nodes. They might be sent at any time during the talk interval, and only include the amount of time the talk interval of the parent node has to be shifted. As schedules are local, nodes only have to coordinate with their parent, i.e., they have to know the wakeup time of their parent, and use it as a basis for establishing schedules with their children.

As beacon messages are critical for correctness, ASLEEP also includes mechanisms to: $i$ ) increase the probability of successful transmission of direct beacons; and

\footnotetext{
${ }^{2}$ It is worthwhile noting that the expected talk interval cannot be lower than one slot. This guarantees that any child has always a chance to send messages to its parent, even after a phase during which it had no traffic to send.
} 
ii) enforce a correct (even if non-optimal) behaviour of nodes in case they miss a direct beacon. The interested reader can find the description of these mechanisms in [2].

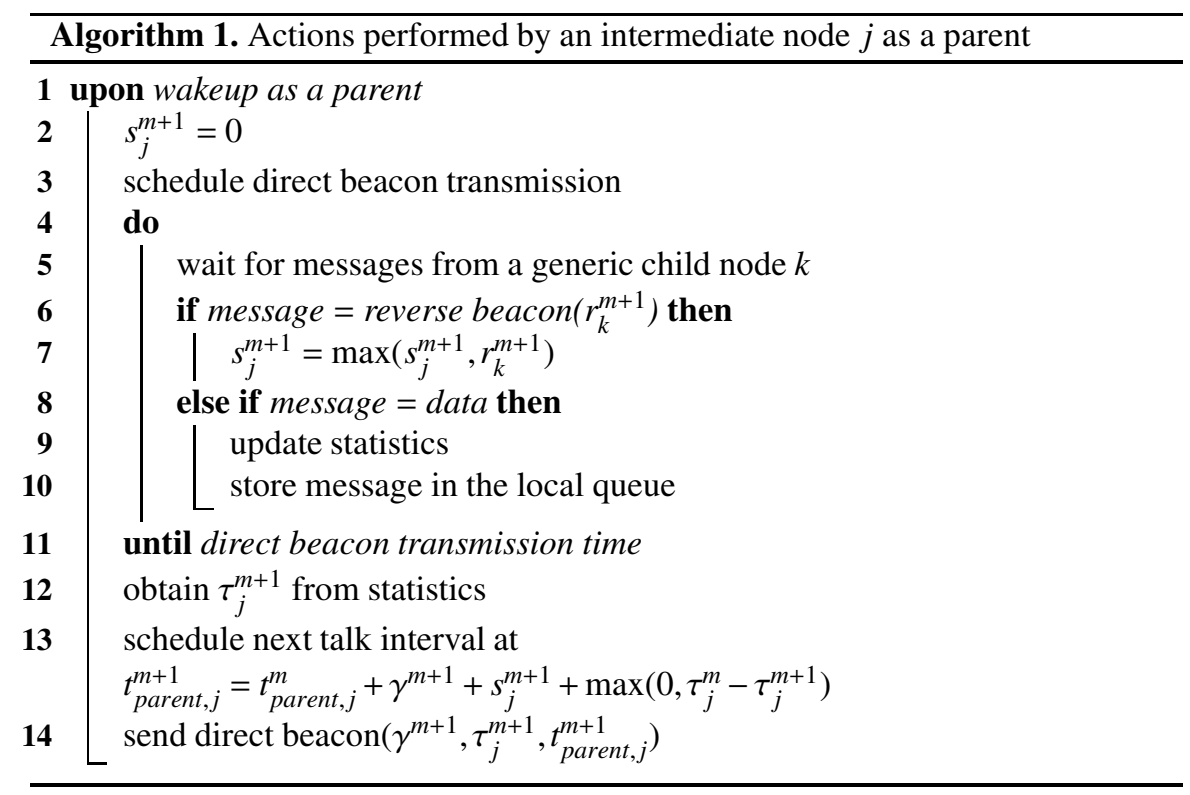

Obviously, the specific actions performed by each single node depend on its position on the routing tree. Algorithm 1 and Algorithm 2 show the actions performed by a generic intermediate node $j$ (child of node $i$ ) during the $m$-th communication period as a father and as a child, respectively 3 . Clearly, the sink node only executes Algorithm 1 while any leaf node only executes Algorithm 2

In the first part of the active interval node $j$ behaves as a parent and talks with its children (Algorithm 1). Upon wakeup, it schedules the direct beacon transmission (line 3). To this end, the expected talk interval estimated in the previous communication period is used. During the talk interval node $j$ receives messages from its children, i.e., data or reverse beacons. Upon receiving a data message, node $j$ updates the statistics on the message inter-reception times and the number of received messages (lines $9+10$ ) that will be later used to estimate the next talk interval. Upon receiving a reverse beacon from a child node $k$, node $j$ realizes that node $k$ requested it to shift its talk interval by the quantity $r_{k}^{m+1}$ included in the reverse beacon (line 6 ). In case of multiple shift requests from the children, the maximum shift value is considered (line 7). Then, node $j$ estimates the duration of the talk interval for the next communication period, $\tau_{j}^{m+1}$ (line 12) — based on the obtained statistics and according to the algorithm described in Section 16.4.2 — and derives the starting time of the next talk interval (line 13). The latter is obtained from the duration of the next

\footnotetext{
${ }^{3}$ In the following, we describe ASLEEP operations in steady state conditions. The initial schedule is established through a special startup phase, not provided here for the sake of space.
} 
communication period $\left(\gamma^{m+1}\right)$, the maximum shift requested by child nodes $\left(s_{j}^{m+1}\right)$ and the difference between the current and estimated next talk interval. Finally, node $j$ broadcasts the direct beacon with the new schedule parameters to its children (line 14. This concludes the talk interval with the children.

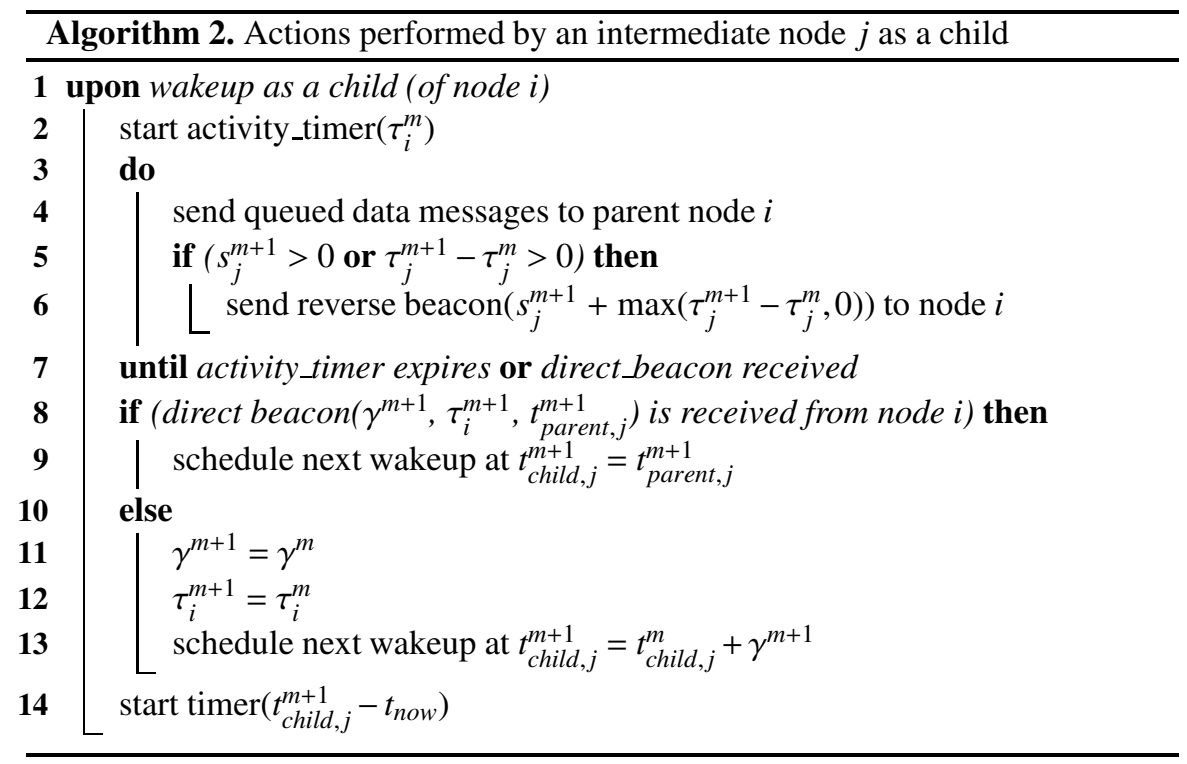

Then, node $j$ acts as a child of node $i$ (Algorithm 2). First of all, node $j$ schedules the end of the talk interval with its parent (line 2). Then, it sends all queued messages (line 4 ) and, if needed, a reverse beacon (lines 5, 6). When sending the reverse beacon, the intermediate node adds to the maximum shift requested by the children (lines 67 7 of Algorithm 1) its own shift $\tau_{j}^{m+1}-\tau_{j}^{m}$, if greater than zero (line 6). Upon receiving the direct beacon from the parent node $i$, node $j$ gets the parameters for the next communication period (lines 8 9). If the direct beacon is missed, then node $j$ uses the current parameters also in the next communication period (lines 11 13). In any case, it sets the timer and enters the sleep mode (line 14).

It can be formally proved that, whenever a change has occurred in the talk interval of one or more nodes with the corresponding children, the global network is able to reach a new coordinated and energy-efficient schedule, under the assumptions that: i) the clocks of nodes are synchronized; and ii) direct and reverse beacons never get lost [2]. Clock synchronization can be achieved through any available clock synchronization protocol [25], and is mitigated by the fact that ASLEEP does not require tight synchronization (values in the order of a few milliseconds are fine). Assumption iil) is rather strong and unlikely to hold in practice since beacons can get lost due to transmission errors and/or collisions. However, thanks to mechanisms for beacon protection and schedule prediction, ASLEEP has been shown to be effective even in environments with high message loss rate [4]. 


\subsection{Protocol Implementation and Experimental Testbed}

We implemented ASLEEP on Tmote Sky [28] sensor nodes with TinyOS 1.1.15 operating system [7]. The Tmote Sky platform is equipped with the Chipcon CC2420 radio transceiver, which is compliant to the 2.4 GHz IEEE 802.15.4 physical layer and has a maximum bit rate of $250 \mathrm{Kbps}$. Our protocol used the default CSMA/CA MAC protocol shipped with TinyOS.

According to the case study considered in the project, the experiments were carried out in a vineyard. For convenience, we performed our experiments in a vineyard located in the Chianti area in Tuscany. Of course, the obtained results are general and do not depend on the specific vineyard. To perform experimental measurements we deployed a testbed WSN consisting of 15 sensor nodes in the vineyard (see Fig. 16.2 $\mathrm{k}$ and Fig. 16.2 b). Nodes were placed at about $50 \mathrm{~cm}$ from the ground.

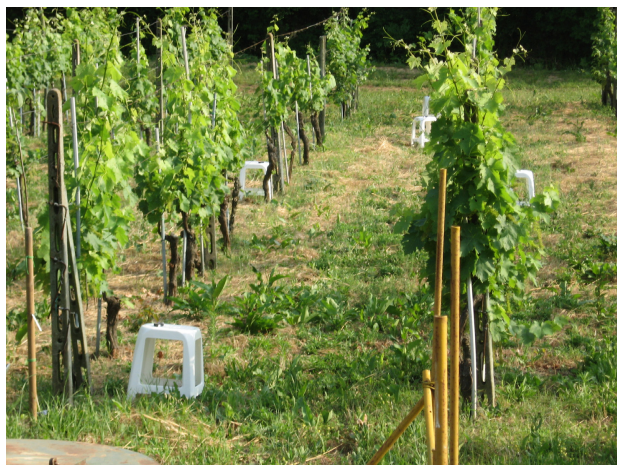

(a)

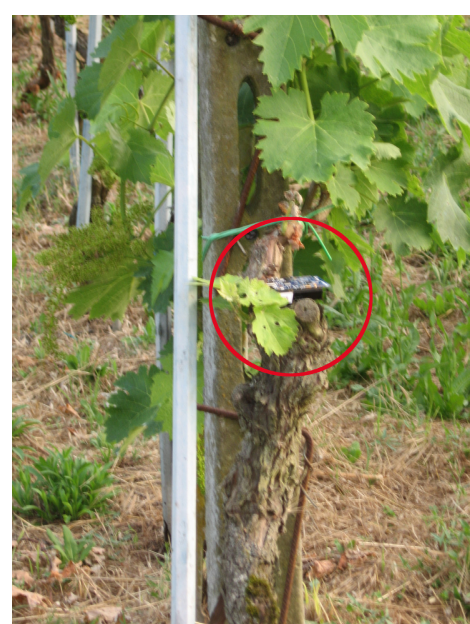

(b)

Fig. 16.2 Experiments site: the vineyard in Vinci (Firenze). General (a) and detailed(b) view.

They periodically sampled the external temperature and humidity, and reported the acquired data to the sink node (node 0 ). In our experiments we used a laptop as the sink node, for semplicity. In a real deployment the sink node could be the gateway node designed in the previous chapter (Chapter 15), which, then, transmits data to a remote central room. Before starting ASLEEP, we performed an initial time synchronization by using a modified version of the scheme in [21], and built the routing tree by exploiting the MintRoute protocol [29]. Synchronization and routing tree formation are repeated periodically over time in order to cope with node failures and changes in channel conditions. In our experimental analysis we considered the following three scenarios. 


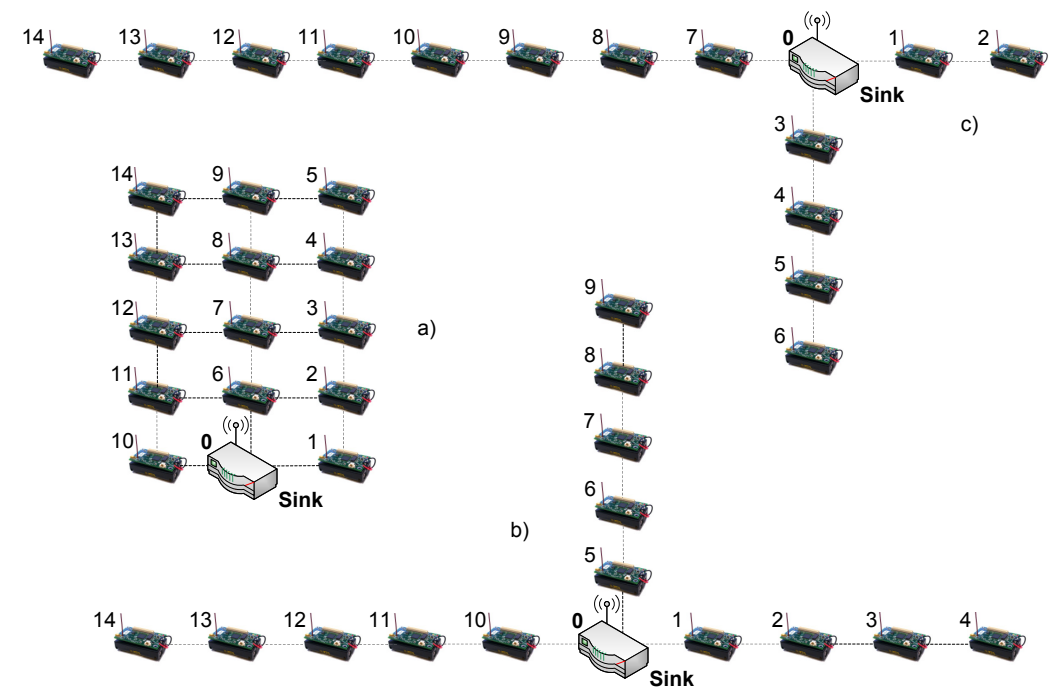

Fig. 16.3 Experimental scenarios: all-in-range (a), multi-hop balanced (b), multi-hop unbalanced (c)

- All-in-Range Scenario. All nodes are within the transmission range of the sink (node 0). Sensor nodes are deployed in the vineyard as shown in Fig. [16.3 $\mathrm{k}$, at a distance of approximately $10 \mathrm{~m}$ from each other, and use the maximum allowed transmission power (so as to reach any other node). Since the transmission range of all sensor nodes is larger than their distance from the sink, a star network topology is expected in this scenario.

- Multi-hop Balanced Scenario. Sensor nodes are deployed in the vineyard to form a T-shaped topology with approximately even branches (see Fig. 16.3b). The transmission power of sensor nodes is set to approximately $-20 \mathrm{dBm}$, with a corresponding transmission range of approximately $12 \mathrm{~m}$. Since the distance between neighbouring nodes deployed along the same row is about $10 \mathrm{~m}$, in theory a multi-hop topology should be formed, with routing sub-trees having almost the same depth.

- Multi-hop Unbalanced Scenario. Sensor nodes are deployed again to form a Tshaped topology, but sub-trees have now different depths $(2,4$, and 8 , as shown Fig. 16.3k). The transmission power and the distance between nodes are the same as in the previous scenario. Therefore, in theory, nodes should form an unbalanced multi-hop topology.

For performance comparison, in addition to ASLEEP, we also implemented the following three sleep scheduling strategies.

- Always-on. In this scheme there is no sleep schedule: nodes never go to sleep and forward messages as soon as they receive them. 
- TAG-like staggered strategy. Sensor nodes use a staggered scheme for sleep coordination, where the talk interval is fixed and equal for all sensor nodes. The talk interval is set to the value of the communication period divided by the depth of the tree, as in TAG [16]. Throughout, we will refer to such a scheme as TAG.

- Optimal fixed staggered scheme. In this scheme the talk interval is fixed and equal for all nodes, as in the TAG scheme. However, the talk interval is approximately equal to the minimum value required in that configuration (this value was derived by preliminary simulation experiments). Throughout, this scheme will be referred to as Fixed.

In a real testbed external conditions change from time to time, sometimes even during the same experiment, and there is no way to control them. In order to improve the statistical accuracy, we replicated each experiment 5 times (each replica was 100 communication-period long). The results presented below are averaged over the entire set of 5 replicas. Standard deviations are also reported.

\subsection{Experimental Results}

In the first part of our analysis we investigated the steady state behaviour of ASLEEP in the three above-mentioned scenarios. We evaluated the performance of the considered protocols in terms of the average duty-cycle of nodes at 1-hop from the sink (the most solicited nodes in terms of energy consumption), the delivery ratio (i.e., fraction of messages delivered to the sink) and the average latency experienced by messages to reach the sink. Table 16.1 shows the parameters used in our experiments. To maintain the experiment duration within reasonable limits, we considered a short communication period (15 s). In a real environment the reporting period is typically much larger, and it is often dictated by the need of maintaining synchronization among nodes. We carried out additional experiments (not presented here) with a communication period of 2 minutes, which substantially confirm the results presented in this section.

When dealing with a real testbed, the actual network topology might be very different than the expected one. This is due to the link quality metrics used for building the data gathering tree [29]. For example, we noted that in the all-in-range scenario, nodes located at the upper corners of the grid (see Fig. 16.3 a) actually associated with an intermediate node in all experiments, resulting in a routing tree of 2 hops. Since the routing tree impacts on performance, we reported in Table 16.2 the (mean and standard deviation of the) routing tree depth observed in the different scenarios.

Fig. 16.4a compares the average duty-cycle of the different sleep/wakeup schemes in the three considered scenarios. ASLEEP outperforms all other schemes as it is able to adjust dynamically the duty-cycle of each sensor node based on its traffic needs, while the other staggered schemes impose the same talk interval to all nodes belonging to the same level of the routing tree. With ASLEEP and Fixed the average duty-cycle increases when passing from the all-in-range, to the multi-hop balanced, and to the multi-hop unbalanced scenario, as expected. With TAG the trend is just the 
Table 16.1 Protocol parameters

\begin{tabular}{ll}
\hline Parameter & Value \\
\hline Communication Period $(\gamma)$ & $15 \mathrm{~s}$ \\
Message rate & $1 \mathrm{msg} / \gamma$ \\
Message size & $28 \mathrm{bytes}$ \\
MAC frame size & $38 \mathrm{bytes}$ \\
Observation window $(L)$ & $10 \gamma$ \\
TI time slot $(q)$ & $150 \mathrm{~ms}$ \\
Beacon Period $(\beta)$ & $60 \mathrm{~ms}$ \\
TI decrease time threshold $(l)$ & $5 \gamma$ \\
TI decrease threshold $(g)$ & $2 q(300 \mathrm{~ms})$ \\
\hline
\end{tabular}

Table 16.2 Routing tree depth in the experiments (mean and standard deviation under parantheses)

\begin{tabular}{llll}
\hline & All-in-range & $\begin{array}{l}\text { Multi-hop } \\
\text { Balanced }\end{array}$ & $\begin{array}{l}\text { Multi-hop } \\
\text { Unbalanced }\end{array}$ \\
\hline ASLEEP & $2.0(0)$ & $3.8(0.45)$ & $5.4(1.34)$ \\
Fixed & $2.0(0)$ & $4.0(0)$ & $4.0(0.71)$ \\
TAG & $2.0(0)$ & $4.0(0)$ & $4.8(1.10)$ \\
Always ON & $2.2(0.45)$ & $3.6(0.55)$ & $4.4(0.55)$ \\
\hline
\end{tabular}

opposite, as in this scheme the length of the talk interval is equal to the communication period divided by the routing tree depth whose average value, for each scenario, is shown in Table 16.2

We now clarify the impact of the average duty-cycle (i.e., energy consumption) on the network lifetime. We consider the model used in [17] and adapt it to the parameters of the Tmote Sky device [28], which is assumed to be powered with a pair of $3000 \mathrm{mAh}$ AA batteries. For a rough estimate, we only consider the contribution of the radio whilst it is in the transmit or receive states (whose current draw is $19.6 \mathrm{~mA}$ ), and neglect all other components (i.e., the sensing and processing subsystems). We also consider a communication period of 2 minutes. In the multi-hop unbalanced scenario, the Always-on scheme achieves a network lifetime of approximately 1 month and a half, which is definitely unsatisfactory for a long-term deployment. TAG extends the network lifetime to about 4 months, which is not yet satisfactory. Fixed allows a lifetime of 600 days (20 months), which is quite good, but it requires to know in advance the talk intervals of sensor nodes, which is clearly unfeasible in practice. Finally, ASLEEP is able to extend the network lifetime to approximately 1200 days (more than three years), thus making long-term deployments actually possible.

Fig. $16.4 \mathrm{~b}$ compares the average message latency introduced by the different sleep/wakeup schemes. Again, ASLEEP outperforms the other staggered schemes. This is because with ASLEEP sensor nodes get shorter active intervals, so that less 


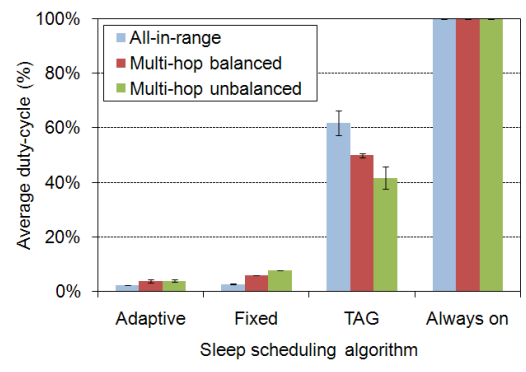

(a)

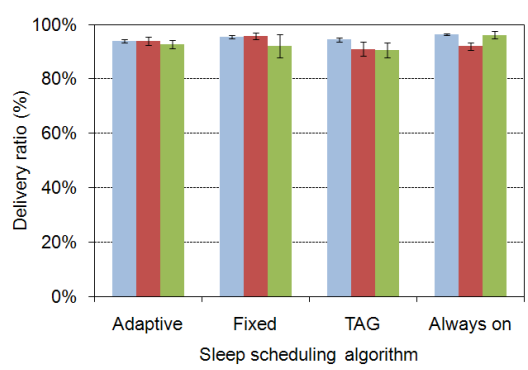

(c)

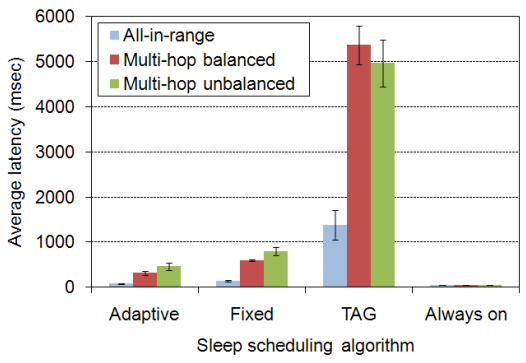

(b)

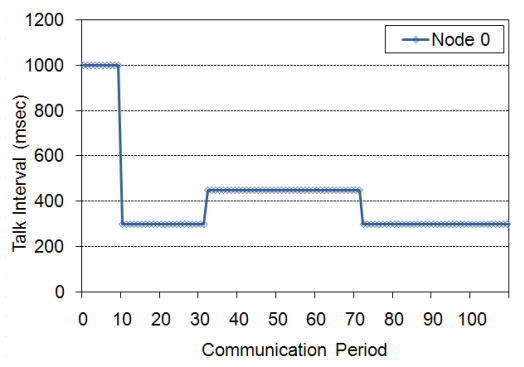

(d)

Fig. 16.4 Performance comparison in the three scenarios in terms of Average Duty-Cycle (a) Average Latency (b) and Delivery Ratio (c) Evolution of the Talk Interval over time (d)

time elapses from when the message is sent by the source to when the same message is received at the sink. In particular, ASLEEP introduces an average latency significantly lower than Fixed (464 ms vs. $798 \mathrm{~ms}$ in the multi-hop unbalanced scenario), and about an order of magnitude lower than TAG.

Finally, Fig. $16.4 \mathrm{k}$ shows the delivery ratio in the three different scenarios. All the sleep/wakeup schemes provide approximately the same delivery ratio (above 90\%), and there is no significant difference when passing from one scenario to another. These results confirm that ASLEEP is robust against possible problems related with schedule exchange and maintenance due to beacon losses.

To conclude our analysis, we also analysed ASLEEP in dynamic conditions. We focused on the multi-hop unbalanced scenario and investigated the effect of a variation in the traffic pattern generated by sensor nodes. Initially all sensor nodes generate 1 message per communication period. After the 30-th communication period the rate increases to 3 messages per communication period and, finally, after the 70-th communication period, it reverts back to the initial value. Fig. $16.4 \mathrm{~d}$ shows the evolution over time of the talk interval shared by the sink and its children as a function of time, in a specific topology (the trend was similar for all other topologies as well). Initially, the talk interval is set to the default value of $1 \mathrm{~s}$. After one observation window $(L=10$ in our experiments) ASLEEP calculates the first estimate and sets the talk interval to 
$300 \mathrm{~ms}$. Then, after the 30-th communication period ASLEEP sets the new talk interval duration to $450 \mathrm{~ms}$. The same value is used up to the 70-th communication period, then it is decreased to $300 \mathrm{~ms}$. Hence, we can see that the talk interval is effectively changed by ASLEEP according to the traffic conditions.

\subsection{Conclusions}

In this chapter we have presented an Adaptive Staggered sLEEp Protocol (ASLEEP) for efficient power management in multi-hop wireless sensor networks (WSNs) for vineyard monitoring applications. The proposed protocol staggers the active periods of nodes according to their position in the routing tree. Unlike traditional staggered schemes, however, the proposed approach can tune the active period of each parent node dynamically, depending on the operating conditions experienced by individual nodes. ASLEEP is thus able to adjust the duty-cycle of sensor nodes to their actual needs, thus minimizing the energy consumption. Finally, ASLEEP is conceived as an independent sleep/wakeup protocol operating above the MAC layer, and can thus be used with any available sensor platform.

We implemented ASLEEP on a real testbed deployed in a vineyard, and performed an extensive experimental analysis. The experimental measurements confirmed the previous very promising simulation results, and showed that a significant reduction in both the sensor nodes duty-cycle and the message latency can be achieved with respect to other staggered approaches where the active periods are fixed and equal for all nodes in the network. Assuming that $i$ ) sensor readings are reported every 2 minutes (which is more than enough for vineyard monitoring); and ii) the energy consumption of the CPU and sensors is negligible with respect to that of the radio, our ASLEEP protocol is able to increase the network lifetime to more than 3 years, thus making a long term WSN deployment actually possible.

\section{References}

1. Akyildiz, I.F., Su, W., Sankarasubramaniam, Y., Cayirci, E.: Wireless Sensor Networks: a Survey. Computer Networks 38(4) (March 2002)

2. Anastasi G., Conti M., Di Francesco M.: An Adaptive Sleep Strategy for Energy Conservation in Wireless Sensor Networks. Technical Report DII-TR-2009-03, University of Pisa, http://info.iet.unipi.it/ anastasi/papers/DII-TR-2009-03.pdf

3. Anastasi, G., Conti, M., Di Francesco, M., Passarella, A.: Energy Conservation in Wireless Sensor Networks: a Survey. Ad hoc Networks 7(3), 537-568 (2009)

4. Anastasi, G., Conti, M., Di Francesco, M.: Extending the Lifetime of Wireless Sensor Networks through Adaptive Sleep. IEEE Transactions on Industrial Informatics 5(3), 351-365 (2009)

5. Baggio, A.: Wireless Sensor Networks in Precision Agriculture. In: Proceedings ACM Workshop on Real-World Wireless Sensor Networks (REALWSN 2005). ACM (2005) 
6. Cao, Q., Abdelzaher, T., He, T., Stankovic, J.: Toward Optimal Sleep Scheduling in Sensor Networks for Rare Event Detection. In: Proc. IPSN 2005 (April 2005)

7. Hill, J., Szewczyk, R., Woo, A., Hollar, S., Culler, D., Pister, K.: System architecture directions for networked sensors. SIGPLAN Not. 35(11), 93-104 (2000)

8. Hohlt, B., Doherty, L., Brewer, E.: Flexible Power Scheduling for Sensor Networks. In: IEEE and ACM International Symposium on Information Processing in Sensor Networks (April 2004)

9. Hohlt, B., Brewer, E.: Network Power Scheduling for TinyOS Applications. In: Proc. IEEE Int'l Conf. on Distributed Computing in Sensor Systems (DCOSS 2006), San Francisco, USA (2006)

10. Intanagonwiwat, C., Govindan, R., Estrin, D.: Directed Diffusion: a Scalable and Robust Communication Paradigm for Sensor Networks. In: Proc. ACM (MobiCOM 2000), Boston, USA (August 2000)

11. Jurdak, R., Baldi, P., Lopes, C.V.: Adaptive Low Power Listening for Wireless Sensor Networks. Transactions on Mobile Computing 6(8), 988-1004 (2007)

12. Keshavarzian, A., Lee, H., Venkatraman, L.: Wakeup Scheduling in Wireless Sensor Networks. In: Proc. ACM MobiHoc 2006, Florence, Italy (May 2006)

13. Li, Y., Ye, W., Heidemann, J.: Energy and Latency Control, in Low Duty-cycle MAC Protocols. In: Proc. IEEE Wireless Communication and Networking Conference, New Orleans, USA (March 2005)

14. Lu, G., Krishnamachari, B., Raghavendra, C.S.: An Adaptive Energy-efficient and Lowlatency Mac for Data Gathering in Wireless Sensor Networks. In: Proc. PDSP 2004 (April 2004)

15. Lu, G., Sadagopan, N., Krishnamachari, B., Goel, A.: Delay Efficient Sleep Scheduling in Wireless Sensor Networks. In: Proc. IEEE Infocom 2005 (March 2005)

16. Madden, S., Franklin, M., Hellerstein, J., Hong, W.: TAG: a Tiny AGgregation Service for Ad-Hoc Sensor Networks. In: Proc. of OSDI (2002)

17. Madden, S.: The Design and Evaluation of a Query Processing Architecture for Sensor Networks. UC Berkeley Ph.D. Thesis (2003)

18. Manjeshwar, A., Agrawal, D.P.: APTEEN: A Hybrid Protocol for Efficient Routing and Comprehensive Information Retrieval in Wireless Sensor Networks. In: Proc. International Workshop on Parallel and Distributed Computing Issues in Wireless Networks and Mobile Computing, Ft. Lauderdale, Florida (April 2002)

19. Mirza, D., Owrang, M., Schurgers, C.: Energy-efficient Wakeup Scheduling for Maximizing Lifetime of IEEE 802.15.4 Networks. In: Proc. International Conference on Wireless Internet (WICON 2005), Budapest (Hungary), pp. 130-137 (July 2005)

20. Paruchuri, V., Basavaraju, S., Kannan, R., Iyengar, S.: Random Asynchronous Wakeup Protocol for Sensor Networks. In: Proc. of BROADNETS 2004 (2004)

21. Ping, S.: Delay Measurement Time Synchronization for Wireless Sensor Networks. IRBTR-03-013, Intel Research Berkeley Lab (2003)

22. Raghunathan, V., Schurgers, C., Park, S., Srivastava, M.B.: Energy Aware Wireless Microsensor Networks. IEEE Signal Processing Magazine 19(2), 40-50 (2002)

23. Rhee, I., Warrier, A., Aia, M., Min, J.: Z-MAC: a Hybrid MAC for Wireless Sensor Networks. In: Proc. ACM SenSys 2005, S. Diego (USA) (November 2005)

24. Schurgers, C., Tsiatsis, V., Srivastava, M.B.: STEM: Topology Management for Energy Efficient Sensor Networks. In: Proc. of the IEEE Aerospace Conference 2002, Big Sky, MT, March 10-15 (2002)

25. Sivrikaya, F., Yener, B.: Time Synchronization in Sensor Networks: A Survey. IEEE Network 18(4), 45-50 (2004) 
26. Sohrabi, K., Gao, J., Ailawadhi, V., Pottie, G.J.: Protocols for Self-organization of a Wireless Sensor Network. IEEE Personal Communications 7(5) (October 2000)

27. TinyDB: a Declarative Database for Sensor Networks, http://telegraph.cs.berkeley.edu/tinydb/

28. Tmote Sky Platform, MoteIV Corporation, http://wwW.sentilla.com/files/pdf/eol/tmote-sky-datasheet.pdf

29. Woo, A., Tong, T., Culler, D.: Taming the underlying challenges of reliable multhop routing in sensor networks. In: Proc. of the 1st ACM Conference on Embedded Networked Sensor Systems (SenSys 2003), Los Angeles, California, pp. 14-27 (November 2003)

30. Yang, X., Vaidya, N.: A Wakeup Scheme for Sensor Networks: Achieving Balance between Energy Saving and End-to-end Delay. In: Proc. of the IEEE Real-Time and Embedded Technology and Applications Symposium (RTAS 2004), pp. 19-26 (2004)

31. Zhang, Z.: Investigation of Wireless Sensor Networks for Precision Agriculture. In: Proceedings 2004 ASABE Annual Meeting. American Society of Agricultural and Biological Engineers (2004)

32. Zheng, R., Hou, J., Sha, L.: Asynchronous Wakeup for Ad Hoc Networks. In: Proc. ACM MobiHoc 2003, Annapolis (USA), June 1-3, pp. 35-45 (2003) 